\title{
Implementation of Professional Pharmacy Services
}

\author{
Abdul Hafeez*, Shmmon Ahmad \\ Department of Pharmaceutics, Glocal School of Pharmacy, Glocal University, Mirzapur Pole, Saharanpur, Uttar Pradesh, India
}

Received October 21, 2020; Revised December 21, 2020; Accepted December 27, 2020

\section{Cite This Paper in the following Citation Styles}

(a): [1] Abdul Hafeez, Shmmon Ahmad, "Implementation of Professional Pharmacy Services," Advances in Pharmacology and Pharmacy, Vol. 8, No. 4, pp. 81 - 86, 2020. DOI: 10.13189/app.2020.080404.

(b): Abdul Hafeez, Shmmon Ahmad (2020). Implementation of Professional Pharmacy Services. Advances in Pharmacology and Pharmacy, 8(4), 81 - 86. DOI: 10.13189/app.2020.080404.

Copyright $\odot 2020$ by authors, all rights reserved. Authors agree that this article remains permanently open access under the terms of the Creative Commons Attribution License 4.0 International License

\begin{abstract}
Quality of pharma-services is defined by the strategies of the pharmacy practices $\&$ the patient outcomes. In order to prevent the disease \& provide the drug therapy to patients, the strategies of pharmacy practices should be enhanced accordingly. The main aim of the pharmacists' professionals is to enhance the quality of the health-care services by adopting the favourable practices. The standards of the services mainly depend upon the provision of the service. Hence, the aim of present research is to identify qualified pharma-service by adopting the service delivery model for community pharmacy. Previously, the pharmacy practices and the standards were described in several ways, for example, analysing the quantitative aspects related with the pharmacy practices, depending upon the strategies of pharmacy practices that allows personal or indirect use of their advanced healthcare expertise, for patients or other healthcare professionals, to improve the treatment cycle with a view to enhancing healthcare outcomes and importance of healthcare. The interpretation of qualified pharmaceutical services integrates operational function principles, method metrics and result steps. A basic computational framework is introduced to integrate all community pharmacy programs. The term can assist in multiple ways including awareness of the broad spectrum of facilities offered by pharma-community, \& promoting establishment of benchmarks for the development and effective delivery of qualified pharmacy services.
\end{abstract}

Keywords Pharma-care, Pharma-services, Pharma-community, Professional Services

\section{Introduction}

There is need to maintain proper relationship among patient \& pharmacist for ensuring that a relationship is developed \& maintained based on integrity, honesty, open interaction, teamwork \& joint decision-making. In this relationship, the pharma-professional protects the individual's health, ensures an appropriate way of treating the consumer's health, $\&$ on behalf of the patients, uses both their professional expertise \& capacities. In return, the individual continues to express personal information $\&$ desires, \& assist in the course of recovery. The pharma-professionals point out plans to ensure individuals undergoing treatment can have access to pharmaceutical medication. Present concepts of pharma-programs do not encompass the whole pharma-community program portfolio because it relies upon identifying programs originating from the prescription treatment term, or are confined to services directly provided by pharmacists as an option. Pharmaceutical treatment is one of the main words used to characterize pharmaceutical practice that reaches into the dispensation sector. The pharmacy treatment concept has been converted into a service-based framework under which medication-related facilities have been delivered by pharmacists "advanced expertise for consumer or health care providers to facilitate efficient and appropriate drug therapy". Subsequent writers changed the concept and developed the word cognitive pharmacy services, which also held pharmacists at the heart, but extended the result to patient wellbeing rather than medication treatment alone, such as "medical services offered through pharma-professionals that utilize its expertise \& experience to have a significant position in contributing to individual's safety via successful engagement"[1].

The word medicinal cognitive services (CPS) is implemented for including elements missing into prior concepts like local government or several health programs 
\& understanding position of another pharma-professional, "CPS is defined as a variety of health initiatives (including prescription care) aimed at enhancing patient safety and the standard of medication therapy facilitated by pharmacy workers". Although medicinal treatment may maintain the central objective of physician-driven programs, there is a requirement for a broader concept to consider wider aspects individually played in healthcare by network of pharma-community. Definitions of pharmaceutical treatment have in several respects limited the function of pharma-community since they concentrate mainly upon medication protection, positiveness\& improving well-being consequences resulting from prescriptions[2].

In past years, both the position of pharmacists as well as the community pharmacy's professional and business activities have evolved and grown. Pharmacists diversify the environments in which they work and specializations are emerging. Around the same period, retail pharmacies integrate specialized pharmacy facilities into both their clinical practice and business model. The continuing growth and distinction of the position of pharmacists is obviously beneficial professionally[3].

Similarly, Community pharmacy services' differentiation and expansion are important to life. It is especially apparent in the new foreign setting, where the conventional business framework is placed under economic strain. A growing phenomenon globally is for states, health care providers and/or consumers able to pay for a variety of pharmaceutical facilities aimed at leading to improved medical results. The widely agreed concept does not occur in pharmacy research papers addressing the wide spectrum of Public Pharmacy practices, facilities and initiatives. A systematic literature review was performed using electronic repositories (MEDLINE, PubMed, without limits of date), document \&Variety of communication to describe the scope of appropriate distribution of pharmaceutical care together with table of contents. The analysis found several terminology and concepts utilized for defining pharma-programs, as well as facets of pharmaceutical practice and service provision, but none represented the complete spectrum of community pharma-services[4].

A broader concept of community-based pharmacy-centric operation is needed because community pharmacies offer an open distribution network that provides a wider variety of clinical resources and a wider spectrum of health care practitioners than is commonly accepted. When community pharmacy evolves into a service company model, a wider concept would aid in investigating the styles and maximum effect of the efficiency, output and delivery of operation. Finally, a wider view would facilitate despite the important role of community pharmacy by the community, government and stakeholders, \& its value offer better in form of medical-care association.

\section{Literature Review}

Based on a detailed assessment of the patient and his or her diagnosis or disorder and recovery, the pharmacist may establish an outcome-oriented opioid therapy strategy with individuals undergoing treatment and with another medical-professionals if appropriate. Procedure may have multiple elements that discuss the illness or disorder of the patient. The pharmacist must closely evaluate the psycho-social dimensions of the condition in developing the prescription, as well as the possible interaction between the expense and/or difficulty of treatment and commitment of patients. As one of the supporters of the hospital, the pharmacist ensures opioid delivery communication between the other service professionals of the pharmacy and the family. Furthermore, the patient must be aware of different pros and cons (i.e., expense, side effects, various control aspects, etc.) of the medication therapy choices \& occasions when one alternative might be more effective depending on the clinical judgement of the pharmacist. The basic aspects of the procedure, and the duties of the provider, must be clarified to the provider thoroughly and in detail. Knowledge should be supplied to the patient at a level which the patient understands. To obtain an overview of the broad spectrum of skilled pharmacy programs which are or may be offered through pharma-community, the following definition is suggested: A pharma-professional service is an operation or set of activities conducted or organised by a pharmacy, administered by a pharma-professional or other health care provider, who directly applies their advanced health expertise Healthcare practitioners, to improve the care cycle with the goal of enhancing health quality and the importance of healthcare[5].

The definition of a quality pharmaceutical provider is built narratively across the framework of Donabedian to establish the level of health-care. Constituents are the pharmacy environment / services in the form of a qualified pharmacy operation, - configuration, \& customer practices of treatment cycle, and patient results. The safety effects are used mainly As the overall measure of medical-care, including technical \& environmental activities that surrounds facilities are among modifiable elements for enhancement[6].

The main components that come under this category are: 1. The Oxford dictionary defines 'function' as an activity, collection of actions, or a sequence of specified operations, synonymously called a plan. The emphasis is on how much interest, gain or enjoyment a company provides. 2 . A collective 'pharmacy' is a recognized concept, authorized and licensed based on the laws and regulations of particular countries. Such framework offers power, control, \&assessing treatment cycle and includes administrative processes, services, staff, and equipment. Such elements may be helpful when evaluating a company. 
To define a company as a qualified pharmaceutical operation, that should be "taken into or arranged through pharma-division" \& conducted accordingly. Whatever the place, When the company suits the medical experience of a pharmacy which requires the pharmacy Expertise and/or abilities of the pharmaceutical system and/or personnel merit becoming a qualified "pharmaceutical" company. A specific example will be a drug delivery program, where such company would be delivered either on-site or off-site, e.g. in an aged care unit or in the home of a customer[7].

Another factor of how programs may be expanded is wellness outreach events, such as a pharmacist presenting an informative lecture to a childcare centre's guardians, or prescribing procedures to a medical centre worker. A licenced pharma-service should be linked to a pharmaceutical sector but that wasn't restricted to the pharma-premises being operated, according to the concepts suggested so far. A registered pharmacy facility is not limited to being run by a pharma-professional but could also be managed by any medical-care provider (Fig. 1). Through incorporating other healthcare providers to the concept, a specialist pharmacy provider might involve a prominent vaccine registered nurse (such as a flu hospital) \& baby therapy network, or a nutritionist conducting a weight reduction campaign or delivering diabetes nutrition education. The description begs the question whether the constitutional \& operational responsibility for every approved pharma-facility actually lies with the operators of the facility \& those on which technical duty lies.

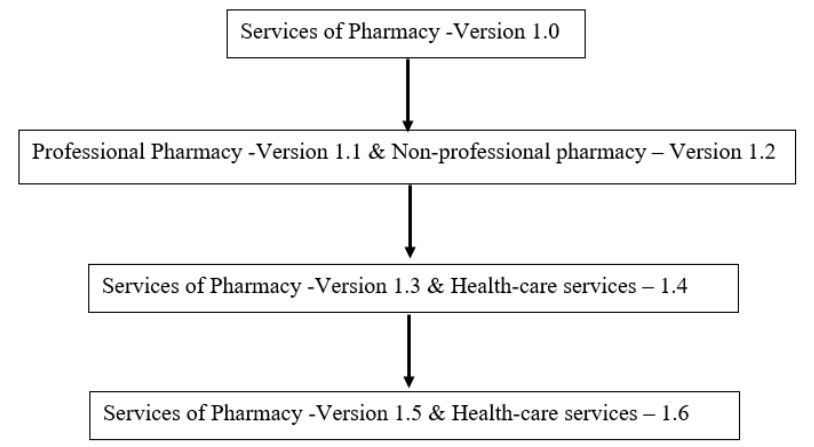

Figure 1. Model of pharmacy provision

\section{Pharmacy Service Provision Model:}

Professional pharmaceutical programs integrate into a pharmacy's general service portfolio (Fig1). The community pharmacy offers a broad range of prescription programs, both qualified and non-skilled. Unlike professional services, the services provided by those who were not professionals don't include implementation of health-care practices, don't enhance pharma-strategies nor do they involve "optimizing the care process" nor are aimed at enhancing patient conditions \& the importance of health insurance. Research on pharma-practice has focused largely over pharma-services (Pharmacist Services 1.3)[8].

Many health care professionals can provide a pharmacy to offer treatment and these will be included in the pharmacies medical program (Other Healthcare Practitioner Services 1.4). Within effort for translating philosophy of pharma-treatment into reality, a significant combination of theories, concepts, meanings, facilities and classification systems has been developed, with little clear logical foundation. The above combination can be listed as Pharma-services (1.3). Pharma-services are spitted in Pharma-services (1.5), (those related to medical treatment which include medication management services, pharmaceutical care services, medical and cognitive pharma-services), \& alike (like health promotion). The Pharmacists' primary emphasis must be on their Specialty field - drug rehabilitation, but all programs which utilize their diversified technique\& information for enhancing health care performance should be recognized (1.1)[1].

Services associated with pharma-professionals should include applying "specialized knowledge in health" of pharma-professional. It would be optimal to have the specialist knowledge of health implemented with the help of evidentiary method \& was an integrated section of treatment. Crucially, that technical expertise isn't confined to drugs. Pharma-professionals are pharmacy experts, and they had extensive health expertise \& several care providers now practise in few applied areas. For example, the detection, diagnosis or triage of health-related issues is a vital component in successful pharmacy practice. In using and clarifying the term "technical" in the description, they are essentially removing facilities not of a skilled nature. Examples involve the uploading or distribution of drugs to patients' houses, ear ringing, picture editing, cosmetic facilities and the selling of goods without the help/suggestion. Such programs don't utilize advanced health-relevant information currently offered through pharma-professionals \& were thus to be called a form of pharma-service, which may remain beyond the reach of a qualified pharma-service[9].

There were several service deliveries, and therefore it would not suffice for enabling direct communication with patient / customer experiences. An extension of concept often responds to pharmacy criticism for relying exclusively on the particular customer. A licenced pharma-service is guided to a customer, an entity, or any other person representing the individual who is suffering from any disease, another medical-care provider, or a society or organisation, or Directly alludes to the direct relation among patient \& worker working in the health-care sector, public, or medical-care professional, while an advisor provides indirect information.[10].

Medical assistance involves monitored pharma or technology employees, including an automated system or a website. Throughout all cases, the knowledge of an intermediary comes from the health professional, who 
accepts responsibility for the treatment plan provided, that is under the supervision or evaluation. A qualified / trained member of medical practitioners would fit with other healthcare professional. Case studies of pharma-technician services rendered by indirect workers involve blood-pressure testing, fabric volume fitting, \& weight management. Descriptions of technologies involve web-links that offer knowledge about well-being.

A key feature of a pharma-engineering service is optimising care. The healthcare method consists of three attitudes \& behaviours, health insurer \& consumer, which intersect type of assistance involvement to achieve results for the wellbeing. The care process on its own includes 3 elements; identification of a necessity (such as preventive measures), use of the facility \& modification of needs. Operations for clients (such as individuals undergoing treatment, care-givers \& stakeholders) involve acknowledgement of requirement, determination for pursuing facility, method of finding treatment \& performing the position associated with ill to sustain treatment[11].

Provider behaviour patterns (including liaison to pharma-professionals) involve acknowledgement, \& diagnosis\& ability to decide the treatment procedure. A goal is to enable the trained pharma-service to be used efficiently by altering any of the behaviours or elements in the treatment cycle. A skilled pharmaceutical service's ultimate goal is to "improve patient results" (prevent negative outcomes, sustain good or optimize fiscal, scientific or humanistic outcomes) and "increase patient care benefit" (maximize health results for dollars which the patient has spent).

Processes in pharmacy together with skilled and company measures treatment habits (client and provider) and results of patient safety are markers of wellbeing and can be assessed to theoretically forecast outcomes. Quality is also used to mean procedure metrics like adherence to the criteria for evidence-based treatment, whereas clinical results are also used as parameters for protection and efficacy. Worth, a fairly recent idea in healthcare, is calculated for a single illness on the average medical care continuum, typically including various locations, facilities and outcome-related costs. Improving efficiency and growing healthcare interest will propel change, moving emphasis from quantity to importance, but health system complexity renders calculating the benefit challenging. Measuring the mechanism and results remains a must.

A variety of hypotheses, models and structures were suggested to explain the difficulty of application in different contexts, including pharmacy. Models of 'flow' are used to explain or direct the method of turning work into reality through different stages of implementation. These typically include evaluation (which includes evaluating the program and atmosphere for the service's introduction, ending with the choice to accept or refuse it), planning or deployment (which includes training the pharma-professionals for delivering facilities).

"Constructions", "Practical Determinants", "Factors", "Determinants of the practice" "implementation", "facilitators" "barriers", or "enablers" are also interchangeably used. Enablers and barriers have also been proposed to be a fluid term, because they may with respect to the barriers \& other aspects beneficial for medical-care. Experts, clinicians \& decision leaders tend to use a range of terms to refer to common ideas, or sometimes identical jargon to describe specific procedures. This also contributes to misinterpretation and uncertainty, which hinders study which experience outcomes across studies and disciplines from being comparable.

The healthcare industry is the lowest category comprising of many delivery variables directly relevant to the process to be applied (e.g. business design, sophistication, recruiting of staff, etc.). The pharmacy personnel environment contains numerous considerations relevant to the delivery of the program by person or community of practitioners (e.g. inspiration, expertise, self-efficacy, and experience, etc.). A number of design considerations related to the environment in which the program is applied are protected by the pharmacy context (e.g. coordination, workforce, history, workflow etc.).

The next stage covers considerations relevant to application of the pharmacy's geographic setting (e.g. customer preferences, coordination with other healthcare practitioners, etc.). Lastly, the framework includes external context-related development considerations (e.g. law, regulation, economic environment, etc.). At any domain point, such implementation variables will manifest as an obstacle (i.e. negative results \& procedure moderator) or even easier methods (i.e. positive results and process optimizer). An illustration to explain this definition is the "time" element for implementation. Within present situation, availability of adequate period to execute the program is a facilitator, wherein shortage of time may be a bottleneck impeding execution of project. The conventional solution to solve this issue has often been based on seeking approaches to increasing the time allocated for service delivery[12].

Most research aimed at identifying factors of implementation, i.e. facilitators and barriers, which contribute to evidence of gap in pharmacy practice. Service providers typically explain these variables, which define them during the distribution or deployment phase of a program. Implementation variables were typically analysed in a straightforward fashion by independently defining them and then documenting their importance with minimal review according to the pharmacy's implementation level. Moreover, and most critically, the reasons and interrelations between them are not usually taken into account[4].

Implementation factors, however, appear to interrelate through mechanisms of "effect and cause" which may vary over the process and stages of implementation. As 
such they can be analysed in a systematic way. Understanding and identifying such causal and complex relationships among different factors of implementation is a major procedure among facilities being implemented. Within this prior case, a number of several applications considerations, like leadership (i.e. engagement, participation \& obligation of the pharma-manager / leaders to introduce service) may affect "time"[13].

Hence, the causes that contribute to short period barrier may be many, such as weak leadership to adapt facility, Operation is not a requirement or inadequate layout or several other operations. If the obstacle is to be tackled as part of an action plan, the implemented solutions will be based on the defined triggers with tailor-made approaches to overcome them, and not on the obstacle as an individual factor. Such weak interpretation of causality generally contributes to overly simple methods in execution directed at addressing the impact (i.e. short period), excluding triggers (i.e. weak leadership, sufficient workflow, or motivation)[14].

Intensive treatments are defined as "rising importance to support current medical care and any premises for opposing real initiative" after review. In the "moment" instance below, tailored strategies of include-naming an inner pharmaceutical champion to act as a catalyst in execution; communicating the urgency of providing the service to pharmacy employees through regular staff processes to discuss target control and rearranging the mechanism to free the facilities from such pharmacy tasks to promote service provision. Subsequently, barriers \& the underlying triggers are established, tailored approaches may be built for the implementation. To address the challenge of successful execution, representing its multi-factorial complexity, suitable multi-faceted approaches are required[3].

\section{Conclusions}

From the present review, it is concluded that there is need to enhance and adopt the pharmacy practices in order to obtain positive patient outcomes. The standard of service must be set in such a way that the patient should be treated in an effective manner without any medical-errors. The proposed pharmacy strategies facilitate the significance of the pharma-community in the form of integrated aspect of the healthcare services as well as a collaborative network. Adoption of this practices improve the skills of the pharmacists. This would help us assess the community pharmacy network with regards to the benefit generated for the medical facilities. Pharmacies are evaluated individually on its average cost \& scope of service delivery, \& price of service delivery. An integrated framework has proved to be beneficial in enhancing the pharmacy practices and the models suggested in this paper have represented the correlation between the Pharmacy \& Non-pharmacy services.
Processes in pharmacy together with skilled and company measures treatment procedures and results of patient safety are markers of wellbeing and can be assessed to theoretically forecast outcomes. Quality is also used to mean procedure metrics like adherence to the criteria for evidence-based treatment, whereas clinical results are also used as parameters for protection and efficacy. If they have effectively incorporated skilled pharmacy, a pharma-community of pharmacies could be known as medical service providers. Pharmacies should be able to discriminate between the amount, form, degree or standard of services they deliver, as part of their total bid, that is, the sum of all services or combinations available to customers. The end aim is to better identify community pharmacy as providers not just of goods but also of qualified pharma-services.

\section{REFERENCES}

[1] J. C. Moullin, D. Sabater-Hernández, F. Fernandez-Llimos, and S. I. Benrimoj, "Defining professional pharmacy services in community pharmacy," Res. Soc. Adm. Pharm., 2013.

[2] J. C. Moullin, D. Sabater-Hernández, and S. I. Benrimoj, "Model for the evaluation of implementation programs and professional pharmacy services," Research in Social and Administrative Pharmacy. 2016.

[3] J. C. Moullin, D. Sabater-Hernández, and S. I. Benrimoj, "Qualitative study on the implementation of professional pharmacy services in Australian community pharmacies using framework analysis,” BMC Health Serv. Res., 2016.

[4] V. Garcia-Cardenas, B. Perez-Escamilla, F. Fernandez-Llimos, and S. I. Benrimoj, "The complexity of implementation factors in professional pharmacy services," Research in Social and Administrative Pharmacy. 2018.

[5] S. Liu et al., "Providing pharmacy services during the coronavirus pandemic," International Journal of Clinical Pharmacy. 2020.

[6] C. Crespo-Gonzalez, V. Garcia-Cardenas, and S. I. Benrimoj, "The next phase in professional services research: From implementation to sustainability," Research in Social and Administrative Pharmacy. 2017.

[7] M. Rosenthal, N. W. Tsao, R. T. Tsuyuki, and C. A. Marra, "Identifying relationships between the professional culture of pharmacy, pharmacists' personality traits, and the provision of advanced pharmacy services," Res. Soc. Adm. Pharm., 2016.

[8] F. Rennung, C. T. Luminosu, and A. Draghici, "Service Provision in the Framework of Industry 4.0," Procedia - Soc. Behav. Sci., 2016.

[9] P. Naik-Panvelkar, C. Armour, J. M. Rose, and B. Saini, "Patient preferences for community pharmacy asthma services: A discrete choice experiment," Pharmacoeconomi cs, 2012 
[10] F. Bradley, D. M. Ashcroft, and P. R. Noyce, "Integration and differentiation: A conceptual model ofgeneral practitioner and community pharmacist collaboration," Res. Soc. Adm. Pharm., 2012.

[11] L. White and C. Klinner, "Service quality in community pharmacy: An exploration of determinants," Res. Soc. Adm. Pharm., 2012.

[12] I. Brazinha and F. Fernandez-Llimos, "Barriers to the implementation of advanced clinical pharmacy services at Portuguese hospitals,” Int. J. Clin. Pharm., 2014.

[13] J. C. Moullin, "Implementation science," in Encyclopedia of Pharmacy Practice and Clinical Pharmacy, 2019.

[14] H. L. Hattingh, F. Kelly, J. Fowler, and A. J. Wheeler, "Implementation of a mental health medication management intervention in Australian community pharmacies: Facilitators and challenges," Res. Soc. Adm. Pharm., 2017.

[15] Venkateswaran, N. "Logistics Information System (Lis) at Pharma Firm-An Evaluation." International Journal of Business and General Management (IJBGM) 7.3 (2018):11-18

[16] Alsamydai, A. M. J., and Ahmed Basim Mohammed Baqer. "Measuring patient satisfaction regarding the quality of healthcare service provided by pharmacists." International Journal of Medicine and Pharmaceutical Science (IJMPS) 5.6 (2015): 71-84.

[17] Alsamydai, Ali Mahmood Jasim. "The Opinions of Pharmacists About The Possibility of Using Electronic Means of Communication by Pharmaceutical Companies to Facilitate The Process of Trade Exchange." International Journal of Business Management \& Research (IJBMR) 4.6 (2014):63-72

[18] Martin, Jennifer, and P. Vijaya Banu. "A Study on the Performance of Health Care Service Industry in Tamil Nadu." International Journal of Human Resource Management and Research (IJHRMR) 8.6 (2018):171-176

[19] Naikwade, Shital, R. Gopal, and Nitin Sippy. "A Study on Serving Class Personnel's Attitude Towards Allopathic or Ayurvedic System of Medicine for Managing Lifestyle Disorder Wrt Panvel, Kalamboli, Kharghar." International Journal of Business Management \& Research (IJBMR) 6.1 (2016):47-56

[20] Si, Ferianto S., and Mahardhika Berliandaldo Se. "Technology Transfer Management in Pharmaceutical Industry Through Freedom to Operate (Fto) Utilization." International Journal of Business Management \& Research (IJBMR) 7.2 (2017):59-66 